\title{
INVESTIGATION OF ELECTRIC GENERATOR ROBUST ALGORITHM UNDER MEASUREMENT NOISES
}

\author{
Igor B. Furtat \\ Lab of Control of Complex systems \\ IPME RAS \\ Russian Federation \\ cainenash@mail.ru
}

\author{
Artem N. Nekhoroshikh \\ Lab of Control of Complex systems \\ IPME RAS \\ Russian Federation \\ becks94@mail.ru
}

Article history:

Received 01.10.18, Accepted 19.12.2018

\begin{abstract}
The paper considers the investigation of a novel robust control algorithm of an electric generator with unknown parameters under bounded disturbances and high-frequency measurement noises. It is assumed that only the load angle is available for measurement, but not the rotor speed. The electric generator model is described by a system of third-order nonlinear differential equations with algebraic coupling ones. The proposed algorithm consisting of static and dynamical terms is based on the separation of the filtering and estimating properties. Differently from existing results the proposed scheme provides the opportunity to control independently the quality of filtering and stabilization. Investigations show that the proposed algorithm attenuates parametric uncertainties and disturbances with accuracy that can be reduced by tuning algorithm parameters.
\end{abstract}

\section{Key words}

Electric generator, measurement noise, disturbance, filter, disturbance attenuation

\section{List of Symbols}

In the paper the following notations are used: $\Delta \delta(t)=\delta(t)-\delta_{0}$;

$\delta(t)$ : the power angle of the generator;

$\delta_{0}$ : the power angle of the generator at the operating point;

$\Delta \omega(t)=\omega(t)-\omega_{0} ;$

$\omega(t)$ : the relative speed of the generator;

$\omega_{0}$ : the synchronous machine speed;

$\Delta P_{e}(t)=P_{e}(t)-P_{m}$;

$P_{e}(t)$ : the active electrical power delivered by the gen- erator;

$P_{m}$ : the mechanical input power;

$D$ : the per unit damping constant;

$H$ : the per unit inertia constant;

$E_{q}^{\prime}(t)$ : the transient EMF in the quadrature axis;

$E_{d}^{\prime}(t)$ : the transient EMF in the direct axis;

$E(t)$ : the EMF in the quadrature axis;

$E_{f}(t)$ : the equivalent EMF in the excitation coil;

$u_{f}(t)$ : the input of the SCR amplifier of the generator;

$T_{d 0}^{\prime}$ : the direct axis transient short circuit time constant;

$k_{c}$ : the gain of the excitation amplifier;

$V_{t}(t)$ : the generator terminal voltage;

$I_{d}(t)$ : the direct axis current;

$I_{q}(t)$ : the quadrature axis current;

$Q_{e}(t):$ the reactive power;

$V_{s}$ : the infinite bus voltage;

$x_{d s}=x_{T}+0,5 x_{L}+x_{d}$;

$x_{q s}=x_{T}+0,5 x_{L}+x_{q}$;

$x_{d s}^{\prime}=x_{T}+0,5 x_{L}+x_{d}^{\prime}$;

$x_{T}$ : the reactance of the transformer;

$x_{L}$ : the reactance of one transmission line;

$x_{d}$ : the direct axis reactance;

$x_{q}$ : the quadrature axis reactance;

$x_{d}^{\prime}$ : the direct axis transient reactance;

$p=d / d t$ : the differential operator.

\section{Introduction}

Since the demand for electricity is increasing continuously, the present trends of power system engineers are to operate the power systems closer to their stability limits without sacrificing the reliability. The stability of power system is very important for secure system operation because an unsecured system can undergo non-periodic major cascading disturbances, or blackouts, which have serious consequences. The world power grids are experiencing many blackouts in recent years due to a variety of effects, such as lighting, severe storms, equipment failures, etc. Thus im- 
portant parameters, such as effective reactances $x_{L}$ of transmission lines, in an electric power system, especially in a multi-machine network, always have some uncertainties. To overcome the parametric uncertainties, several control schemes have been proposed. For example, robust adaptive backstepping control [Roy, Mahmud, Shen, and Oo, 2015], sliding mode control [Loukianov, Canedo, Fridman, and Soto-Cota, 2011; Munoz-Aguilar, Doria-Cerezo, Fossas, and Cardoner, 2011], passivity-based approach [Furtat et al., 2016; Galaz, Ortega, Bazanella, and Stankovic, 2003; Luo, 2007], model predictive control [Zheng, Zhou, Zhu, Zhang, Li, and Fu, 2016], and feedback linearization approach [Kenne, Goma, Nkwawo, Lagarrigue, Arzande, and Vannier, 2010; Mahmud, Pota and Hossain, 2012].

A robust adaptive backstepping scheme is proposed in [Roy, Mahmud, Shen, and Oo, 2015] to design an excitation controller for the electric generator, which considers both parametric uncertainties and external disturbances along with measurement noises. The performance of the controller degrades if adaptation gains are not selected properly.

In [Loukianov, Canedo, Fridman, and Soto-Cota, 2011; Munoz-Aguilar, Doria-Cerezo, Fossas, and Cardoner, 2011], sliding mode excitation controllers are used to provide robustness against parametric uncertainties and external disturbances. However, the main limitation of the sliding mode excitation controller is the selection of the sliding surface during the fast transients which are very common in power system operations.

Works [Furtat et al., 2016; Galaz, Ortega, Bazanella, and Stankovic, 2003; Luo, 2007] have used the total energy of the system to design the excitation controller for the electric generator. However, it is practically hard and sometime, quite infeasible to calculate the accurate energy of the electric generator, which makes this approach not so popular in power system applications.

Nonlinear model predictive control scheme allows direct design of excitation control for the electric generator while optimizing a nonlinear objective function [Zheng, Zhou, Zhu, Zhang, Li, and Fu, 2016]. This approach is useful for the electric generator application as the objective function is quite simple and straightforward. However, the implementation of the model predictive control scheme requires the exact parametric information of the system in order to achieve the desired control objective and the control performance may degrade if the parameters are uncertain.

The direct feedback linearization scheme is proposed in [Kenne, Goma, Nkwawo, Lagarrigue, Arzande, and Vannier, 2010] which considers only the parametric uncertainties of the electric generator. The linear controller for the feedback linearized system designed is a linear quadratic regulator (LQR). It uses the rotor angle of the generator as a state for its state feedback.
A robust partial feedback linearizing controller is proposed in [Mahmud, Pota and Hossain, 2012] where both state dependent and parametric uncertainties are modeled as a structured uncertainty that is explicitly used in LQR design. It uses the speed deviation signal as the output function. As the designed LQR controller depends on the pre-estimated structured uncertainty, its performance may degrade if the parameter changes are large. Therefore, the existing feedback linearization plus LQR approach does not have the capability to ensure the robustness of the closed-loop system against the variations in the system parameters, external disturbances, and measurement noises.

The review has shown that despite the large amount of results on power system control, the issue of robustness of the excitation controllers to the model uncertainties, external disturbances and measurement noises has not been fully studied and resolved. There is still a pressing need to develop practically useful excitation controllers with good performance and robustness to resolve this important issue.

To provide a solution to the open problem, this paper will develop and investigate a robust controller using artificial delay [Nekhoroshikh and Furtat, 2017] for the excitation control of the electric generator. It is assumed that only the noisy load angle is available for measurement, but not the rotor speed. The proposed control signal consists of statical and dynamical terms. The former compensates static mechanical input power. The latter deals with parametric uncertainties and disturbances. Firstly, low-pass filter is used to filter out the high-frequency components of the noise before feeding the measurement into the observer. Secondly, delayed stabilizing output-feedback is designed using filtered values of the load angle. The proposed control scheme provides the robustness against parametric uncertainties, disturbances, and high-frequency measurement noises. Furthermore, the stability region of the generator could be obtained during control parameter tuning.

\section{Plant Model and Problem Statement}

In this paper an infinite bus model is considered. Thus, the simplified model can be described as a single generator connected through two parallel transmission line to a large network approximated by an infinite bus. According to [Anderson and Fouad, 1977; Bergan, 1986], the electrical generator model connected to infinite bus is described by the following equations

- mechanical equations:

$$
\begin{aligned}
\Delta \dot{\delta}(t) & =\Delta \omega(t) \\
\Delta \dot{\omega}(t) & =-\frac{D}{2 H} \Delta \omega(t)-\frac{\omega_{0}}{2 H} \Delta P_{e}(t)
\end{aligned}
$$


- generator electrical dynamics:

$$
\begin{aligned}
\dot{E}_{q}^{\prime}(t) & =\frac{1}{T_{d 0}^{\prime}}\left[E_{f}(t)-E(t)\right] \\
E_{f}(t) & =k_{c} u_{f}(t)
\end{aligned}
$$

- electrical equations:

$$
\begin{aligned}
E(t) & =\frac{x_{d s}}{x_{d s}^{\prime}} E_{q}^{\prime}(t)-\frac{x_{d}-x_{d}^{\prime}}{x_{d s}^{\prime}} V_{s} \cos \delta(t) \\
E_{d}^{\prime}(t) & =\left(x_{q}-x_{d}^{\prime}\right) I_{q}(t) \\
V_{t}(t) & =\sqrt{\left(E(t)-x_{d} I_{d}(t)\right)^{2}+\left(x_{q} I_{q}(t)\right)^{2}} \\
I_{d}(t) & =\frac{E(t)}{x_{d s}}-\frac{V_{s}}{x_{d s}} \cos \delta(t) \\
I_{q}(t) & =\frac{V_{s}}{x_{q s}} \sin \delta(t) \\
P_{e}(t) & =\frac{V_{s}}{x_{d s}} E(t) \sin \delta(t) \\
& +\frac{V_{s}^{2}}{2}\left(\frac{1}{x_{q s}}-\frac{1}{x_{d s}}\right) \sin (2 \delta(t)) \\
Q_{e}(t) & =\frac{V_{s}}{x_{d s}} E(t) \cos \delta(t)-\frac{V_{s}^{2}}{2}\left(\frac{1}{x_{q s}}+\frac{1}{x_{d s}}\right) \\
& +\frac{V_{s}^{2}}{2}\left(\frac{1}{x_{q s}}-\frac{1}{x_{d s}}\right) \cos (2 \delta(t))
\end{aligned}
$$

We study model (1)-(11) under the following two assumptions.

1. Parameters $D, H, T_{d 0}^{\prime}, k_{c}, x_{T}, x_{L}, x_{d}, x_{q}$ and $x_{d}^{\prime}$ belong to a known compact set $\Xi$.

2. Only noisy load angle

$$
z(t)=\delta(t)+w(t)
$$

is available for measurement, where $w(t)$ is a highfrequency measurement noise.

The proposed control law should ensure the stabilization of the electric generator at the operating point, i.e.

$$
\lim _{t \rightarrow \infty} \sup |\Delta \delta(t)| \leq \epsilon
$$

where $\epsilon>0$ is a required accuracy.

\section{Robust Controller Design and Investigation}

From the section above one can conclude that model of electric generator is essentially nonlinear. To design a linear controller, we can treat all nonlinearities as internal disturbances. To this end, let us rewrite model (1)-(11) as follows

$$
Q(p, t) \Delta \delta(t)=-k \sin \delta(t) u_{f}(t)+\varphi(t),
$$

where

$$
\begin{aligned}
Q(p, t) & =p^{3}+q_{2} p^{2}+q_{1}(t) p ; \\
q_{2} & =\frac{D}{2 H}+\frac{x_{d s}}{x_{d s}^{\prime} T_{d 0}^{\prime}} ; \\
q_{1}(t) & =\frac{D x_{d s}}{2 H x_{d s}^{\prime} T_{d 0}^{\prime}}+\frac{\omega_{0} V_{s}}{2 H x_{d s}^{\prime}} E_{q}^{\prime}(t) \cos \delta(t) \\
& +\frac{\omega_{0} V_{s}^{2}}{2 H}\left(\frac{1}{x_{q s}}-\frac{1}{x_{d s}^{\prime}}\right) \cos (2 \delta(t)) ; \\
k & =\frac{\omega_{0} V_{s} k_{c}}{2 H x_{d s}^{\prime} T_{d 0}^{\prime}} ; \\
\varphi(t) & =\frac{\omega_{0} x_{d s}}{2 H x_{d s}^{\prime} T_{d 0}^{\prime}} P_{m} \\
& -\frac{\omega_{0} x_{d s} V_{s}^{2}}{4 H x_{d s}^{\prime} T_{d 0}^{\prime}}\left(\frac{1}{x_{q s}}-\frac{1}{x_{d s}}\right) \sin (2 \delta(t)) .
\end{aligned}
$$

Since output measurements contain high-frequency noise the signal $z(t)-\delta_{0}$ is passed through a singleinput single-output low-pass filter of the form

$$
\Delta \hat{\delta}(t)=\frac{1}{\prod_{i=1}^{r}\left(\mu \sigma_{i} p+1\right)}\left[z(t)-\delta_{0}\right]
$$

where $\Delta \hat{\delta}(t)$ is the estimate of $\Delta \delta(t), \mu<<1$; $\sigma_{0}, \ldots, \sigma_{r}>0$ are time constants; $r$ is the dynamical order of the filter.

It is worthwhile to mention that the effect of preceding a high-gain observer by a low-pass filter has been analysed in [Khalil and Priess, 2016].

It is a well-known fact that an electric generator can not be stabilized with zero voltage applied to the excitation coil $u_{f}(t)$ and non-zero mechanical input power $P_{m}$. Thus, signal $u_{f}(t)$ has to consist of statical and dynamical terms. The former compensates static mechanical input power. The latter deals with parametric uncertainties and disturbances. So let us define control law in the following form

$$
u_{f}(t)=\frac{E_{0}}{k_{c}}+\alpha \operatorname{sgn}[\sin \hat{\delta}(t)] \sum_{j=0}^{2} d_{j} \Delta \bar{\delta}^{(j)}(t)
$$

where $E_{0}$ is the EMF in the quadrature axis at the operating point; $\alpha>0$ is the adjustable parameter; $d_{0}, d_{1}, d_{2}$ are coefficients chosen such that the polynomial $D(\lambda)=d_{2} \lambda^{2}+d_{1} \lambda+d_{0}$ is Hurwitz; $\hat{\delta}(t)=$ $\Delta \hat{\delta}(t)+\delta_{0}$ is the estimate of power angle; $\Delta \bar{\delta}^{(i)}(t)$ is the estimate of the $i$ th derivative of $\Delta \hat{\delta}(t)$.

According to assumption 2, the derivatives of the output can not be measured directly. Therefore, one can use the finite differences to estimate the derivatives and consequently to implement the control law (16). So the 
following observer is introduced

$$
\begin{aligned}
\Delta \bar{\delta}(t) & =\Delta \hat{\delta}(t), \\
\Delta \bar{\delta}^{(1)}(t) & =\frac{\Delta \bar{\delta}(t)-\Delta \bar{\delta}(t-h)}{h}, \\
\Delta \bar{\delta}^{(2)}(t) & =\frac{\Delta \bar{\delta}^{(1)}(t)-\Delta \bar{\delta}^{(1)}(t-h)}{h},
\end{aligned}
$$

where $h>0$ is the artificial delay.

Substituting (17) in (16), it yields:

$$
u_{f}(t)=\frac{E_{0}}{k_{c}}+\alpha \operatorname{sgn}[\sin \hat{\delta}(t)] \sum_{j=0}^{2} \bar{d}_{j} \Delta \delta(t-j h)
$$

where

$$
\begin{aligned}
& \bar{d}_{0}=\frac{d_{2}+d_{1} h+d_{0} h^{2}}{h^{2}} ; \\
& \bar{d}_{1}=-\frac{2 d_{2}+d_{1} h}{h^{2}} ; \quad \bar{d}_{2}=\frac{d_{2}}{h^{2}} .
\end{aligned}
$$

Since all signals in (15) and (18) are measurable so the control law is simple and practically realizable.

Let us formulate the main result.

Theorem 1. Let assumptions be hold and noise $w(t)$ be bounded. Then there exist coefficients $\alpha>0, \mu>0$, $h>0$ and numbers $0 \leq \delta_{\min } \leq \delta_{\max } \leq \pi$ such that algorithm consisting of filter (15) and control law (18) ensures existence of stability region $\left[\delta_{\min }, \delta_{\max }\right]$.

Proof. Taking into account (16), we investigate the following closed-loop system

$$
\left\{\begin{aligned}
Q(p, t) \Delta \delta(t) & =-\alpha \tilde{k}(t) g(t)+\tilde{\varphi}(t) \\
R(p) \Delta \hat{\delta}(t) & =\Delta \delta(t)+w(t)
\end{aligned}\right.
$$

where $\tilde{k}(t)=k \operatorname{sgn}[\sin \hat{\delta}(t)] \sin \delta(t)$;

$g(t)=\sum_{j=0}^{2} \bar{d}_{j} \Delta \delta(t-j h)$;

$\tilde{\varphi}(t)=\varphi(t)-\left(k E_{0} / k_{c}\right) \sin \delta(t)$;

$R(p)=\prod_{i=1}^{r}\left(\mu \sigma_{i} p+1\right)$.

Introducing filtering error $e=\Delta \delta-\Delta \hat{\delta}$, function $g(t)$ can be represented as follows:

$$
\begin{aligned}
g(t) & =\bar{d}_{0}[\Delta \delta(t)-e(t)] \\
& +\bar{d}_{1}[\Delta \delta(t-h)-e(t-h)] \\
& +\bar{d}_{2}[\Delta \delta(t-2 h)-e(t-2 h)] .
\end{aligned}
$$

We consider a case when the dimension of filter (15) equals one. It follows from (15) that

$$
\Delta \hat{\delta}^{(1)}(t)=-\frac{1}{\mu \sigma_{1}} \Delta \hat{\delta}(t)+\frac{1}{\mu \sigma_{1}}[\Delta \delta(t)+w(t)]
$$

or

$$
e^{(1)}(t)=\Delta \delta^{(1)}(t)-\frac{1}{\mu \sigma_{1}}[e(t)+w(t)]
$$

Therefore, closed-loop system (19) could be rewritten as follows in the form

$$
\left\{\begin{aligned}
\dot{\varepsilon}(t) & =A(t) \varepsilon(t)+F_{1}(t) \varepsilon(t-h) \\
& +F_{2}(t) \varepsilon(t-2 h)+\psi(t) \\
\Delta \delta(t) & =L \varepsilon(t)
\end{aligned}\right.
$$

where

$$
\begin{aligned}
& \varepsilon(t)=\left[\begin{array}{c}
\Delta \delta(t) \\
\Delta \delta^{(1)}(t) \\
\Delta \delta^{(2)}(t) \\
e(t)
\end{array}\right] ; \quad L=\left[\begin{array}{llll}
1 & 0 & 0 & 0
\end{array}\right] \\
& A(t)=\left[\begin{array}{cccc}
0 & 1 & 0 & 0 \\
0 & 0 & 1 & 0 \\
-\alpha \tilde{k}(t) \bar{d}_{0} & -q_{1}(t) & -q_{2} & \alpha \tilde{k}(t) \bar{d}_{0} \\
0 & 1 & 0 & -\frac{1}{\mu \sigma_{1}}
\end{array}\right] \text {; } \\
& F_{j}(t)=-\alpha \tilde{k}(t)\left[\begin{array}{cccc}
0 & 0 & 0 & 0 \\
0 & 0 & 0 & 0 \\
\bar{d}_{j} & 0 & 0 & -\bar{d}_{j} \\
0 & 0 & 0 & 0
\end{array}\right], \quad j=1,2 \text {; } \\
& \psi(t)=\left[\begin{array}{c}
0 \\
0 \\
0 \\
-\frac{1}{\mu \sigma_{1}}
\end{array}\right] w(t)+\left[\begin{array}{l}
0 \\
0 \\
1 \\
0
\end{array}\right] \tilde{\varphi}(t) \text {. }
\end{aligned}
$$

To stability analysis of (23), we consider LyapunovKrasovskii functional in the form

$$
\begin{aligned}
V & =\varepsilon^{T}(t) P \varepsilon(t)+\int_{-h}^{0} \varepsilon^{T}(t+s) N_{1} \varepsilon(t+s) d s \\
& +\int_{-2 h}^{0} \varepsilon^{T}(t+s) N_{2} \varepsilon(t+s) d s
\end{aligned}
$$

where $P=P^{T}$ is a solution of LMI $A^{T}(t) P+$ $P A(t) \leq-Q, Q=Q^{T}>0, N_{j}=N_{j}^{T}>0, j=1,2$.

Remark 1. We note that the matrix $A(t)$ depends on functions $\sin \delta(t), \cos \delta(t)$ and $E_{q}^{\prime}(t)$ that are bounded. Therefore, one have to solve this LMI simultaneously for all $\delta(t)$ in stability region of the generator, i.e. $0 \leq$ $\delta_{\min } \leq \delta(t) \leq \delta_{\max } \leq \pi$, applying the same decision matrix $P$.

Obviously, there exists a solution for $\delta=\pi / 2$. Then LMI could be feasible in vicinity of $\delta=\pi / 2$ also. Therefore, changing value of $\delta$ the stability region $\left[\delta_{\min }, \delta_{\max }\right]$ of generator can be found. 
Taking derivative of (24) w.r.t. $t$, we get

$$
\begin{aligned}
\dot{V} & \leq-\varepsilon^{T}(t)\left[Q-N_{1}-N_{2}\right] \varepsilon(t) \\
& -\sum_{j=1}^{2} \varepsilon^{T}(t-j h) N_{j} \varepsilon(t-j h) \\
& +2 \varepsilon^{T}(t) \sum_{j=1}^{2} F_{j} \varepsilon(t-j h)+2 \varepsilon^{T}(t) P \psi(t) .
\end{aligned}
$$

We estimate terms in (25) as follows

$$
\begin{aligned}
& 2 \varepsilon^{T}(t) F_{j}(t) \varepsilon(t-j h) \leq \chi \varepsilon^{T}(t) P^{2} \varepsilon(t) \\
& +\chi^{-1} \varepsilon^{T}(t-j h) F_{j}^{T}(t) F_{j}(t) \varepsilon(t-j h) ; \\
& 2 \varepsilon^{T}(t) P \psi(t) \leq \chi \varepsilon^{T}(t) P^{2} \varepsilon(t)+\chi^{-1}|\psi(t)|^{2},
\end{aligned}
$$

where $\chi>0$ is a small parameter, $j=1,2$.

Taking into account (26), we rewrite (25) as follows

$$
\begin{aligned}
\dot{V} & \leq-\varepsilon^{T}(t) W \varepsilon(t)-\varepsilon^{T}(t-h) R_{1} \varepsilon(t-h) \\
& -\varepsilon^{T}(t-2 h) R_{2} \varepsilon(t-2 h)+\tau,
\end{aligned}
$$

where $W=Q-N_{1}-N_{2}-3 \chi P^{2}, R_{j} \geq N_{j}-$ $\chi^{-1} F_{j}^{T}(t) F_{j}(t), j=1,2, \tau=\sup _{t \geq 0} \chi^{-1}|\psi(t)|^{2}$. Obviously, there exist parameters $\alpha$ and $\chi$ such that $W>0, R_{1}>0$ and $R_{2}>0$. Thus, inequality (27) can be estimated in the form

$$
\dot{V} \leq-\lambda_{\min }(W) \varepsilon^{T}(t) \varepsilon(t)+\tau,
$$

where $\lambda_{\min }(W)$ is the smallest eigenvalue of $W$. As a result, goal (13) holds.

Other cases depending on the dimension of filter (15) could be proved in the similar manner.

\section{Conclusion}

The paper investigates the robust control problem of an electric generator with unknown parameters under the effect of bounded disturbances and the presence of high-frequency measurement noises. It is assumed that only the load angle is available for measurement. It is a well-known fact that an electric generator can not be stabilized with zero voltage applied to the excitation coil and non-zero mechanical input power. Thus, the proposed control signal consists of statical and dynamical terms. The former compensates static mechanical input power. The latter deals with parametric uncertainties and disturbances.

It is shown that the electric generator is stabilized when coefficient $\mu$ and artificial time delay $h$ are sufficiently small. Unlike the existing results the proposed algorithm allows to increase the required accuracy due to the separation principle of filtering and estimating properties. The control scheme consisting of low-pass filter and delayed stabilizing delayed output feedback is easy in implementation. Furthermore, the stability region of the generator could be obtained during control parameter tuning.

\section{Acknowledgement}

The proof of Theorem was supported by Program No. 30 of Presidium of Russian Academy of Sciences.

\section{References}

Anderson, P.M. and Fouad, A.A. (1977). Power System Control and Stability. Iowa State University Press. Ames, Iowa.

Bergan, A.R. (1986). Power Systems Analysis. Prentice-Hall. New Jersey.

Galaz, M., Ortega, R., Bazanella, A.S., and Stankovic, A.M. (2003). An energy-shaping approach to the design of excitation control of synchronous generators. Automatica, 1, pp. 111-119.

Furtat, I.B. (2015). Robust Static Control Algorithm for Linear Objects. Automation and Remote Control, 76(3), pp. 446-457.

Furtat, I., Tergoev, N., Tomchina, O., Kazi, F, and Singh, N. (2016) Speed-Gradient-Based Control of Power Network: Case Study. Cybernetics and Physics, 5(3)16, pp. 85-90.

Kenne, G., Goma, R., Nkwawo, H., Lagarrigue, F.L., Arzande, A., and Vannier, J,C. (2010). An improved direct feedback linearization technique for transient stability enhancement and voltage regulation of power generators. International Journal of Electrical Power and Energy Systems, 32(7), pp. 809-816.

Khalil, H.K. and Priess, S. (2016). Analysis of the Use of Low-Pass Filters with High-Gain Observers. IFACPapersOnLine, 49(18), pp. 488-492.

Loukianov, A.G., Canedo, J.M., Fridman, L.M., and Soto-Cota, A. (2011). High-Order Block SlidingMode Controller for a Synchronous Generator With an Exciter System. In Proc. IEEE Transactions on Industrial Electronics Jan. 2011. pp. 337-347.

Luo, X.S (2007). Passivity-based adaptive control of chaotic oscillations in power system. Chaos, Solitons \& Fractals 31, 3, pp. 665-671.

Mahmud, M.A., Pota, H.R., and Hossain, M.J. (2012). Robust zero dynamic excitation controller for power systems under structured uncertainty. In Proc. of the 2nd Australian Control Conference (AUCC) Sydney, Australia, Nov. 2012. pp. 100-109.

Munoz-Aguilar, R.S., Doria-Cerezo, A., Fossas, E., and Cardoner, R. (2011). Sliding Mode Control of a Stand-Alone Wound Rotor Synchronous Generator. In Proc. IEEE Transactions on Industrial Electronics Oct. 2011. pp. 4888-4897.

Nekhoroshikh, A.N., and Furtat, I.B. (2017). Ro- 
bust stabilization of linear plants under uncertainties and high-frequency measurement noises. In 25th Mediterranean Conference on Control and Automation, MED 2017 Valletta, Malta, Jul. 2017. pp. 12751280.

Roy, T.K., Mahmud, M.A., Shen, W.X., and Oo, A.M.T. (2015). Robust adaptive backstepping excitation controller design for simple power system models with external disturbances. In Proc. IEEE Conference on Control Applications (CCA) Sydney, NSW, 2015. pp. 715-720.

Zheng, Y., Zhou, J., Zhu, W., Zhang, C., Li, C., and $\mathrm{Fu}$, W. (2016). Design of a multimode intelligent model predictive control strategy for hydroelectric generating unit. Neurocomputing, 207, pp. 287-299. 